\title{
Bi-invariant differential operators on the Euclidean motion group and applications to generalized Radon transforms
}

\author{
Fulton B. Gonzalez
}

\begin{abstract}
We determine the algebra of bi-invariant differential operators (i.e., the center of the universal enveloping algebra) of the group $M(n)$ of rigid motions of $\mathbf{R}^{n}$ by explicitly describing a set of $\left[\frac{1}{2}(n+1)\right]$ algebraically independent generators of orders $2,4,6, \ldots$. Passing to the complexification of the Lie algebra of $M(n)$ we then obtain a description of the algebra of bi-invariant differential operators on the connected Poincaré group $S O_{0}(p, q) \times \mathbf{R}^{p+q}$ (semidirect product). We also apply our main result to show how a certain generalization of the Radon transform, defined on the affine Grassmannian manifold of $p$-dimensional planes in $\mathbb{R}^{n}$, intertwines the $M(n)$-invariant differential operators on such manifolds.
\end{abstract}

\section{Introduction}

For a Lie group $G$ let $\mathbf{D}(G)$ denote the algebra of left invariant differential operators on $G$ and let $\mathbf{Z}(G) \subseteq \mathbf{D}(G)$ denote the algebra of left and right invariant differential operators on $G$. In this paper we determine the algebra $\mathbf{Z}(G)$ when $G$ is the group $M(n)$ of rigid motions of the Euclidean space $\mathbf{R}^{n}$. We will show that $\mathbf{Z}(M(n))$ has $\left[\frac{1}{2}(n+1)\right]$ algebraically independent generators, having orders $2,4,6, \ldots$, and we will describe these generators explicitly.

Passing to the complexification of the Lie algebra of $M(n)$ we then obtain a description of the algebra $\mathbf{Z}(G)$, when $G$ is the semidirect product $S O(n, \mathbf{C}) \times \mathbb{C}^{n}$, and also when $G$ is the general connected Poincaré group $S O_{0}(p, q) \times \mathbf{R}^{p+q}$.

Supported in part by NSF Grant DMS-8601965. 
The problem of describing the algebra of bi-invariant differential operators on the above semidirect products was also considered by S. Takiff [14], but was only completely solved in the case $n \leqq 4$.

Next, let $H$ be any closed subgroup of a Lie group $G$ and let $\mathbf{D}(G / H)$ be the algebra of differential operators on the manifold $G / H$ which are invariant under the action of $G$. If $\pi: G \rightarrow G / H$ is the natural projection, let $\mu: \mathbb{Z}(G) \rightarrow \mathbf{D}(G / H)$ be the homomorphism defined as in [7] by $(\mu(D) f) \circ \pi=D(f \circ \pi)$ for $D \in \mathbb{Z}(G)$ and $f \in C^{\infty}(G / H)$. Setting $G=M(n)$ and $H$ the subgroup leaving a certain $p$-dimensional subspace of $\mathbf{R}^{n}$ invariant, the coset space $G / H$ is then the manifold $G(p, n)$ of $p$-planes in $\mathbf{R}^{n}$. Using the description of $\mathbf{D}(G(p, n))$ in [4], we will show that the map $\mu: \mathbf{Z}(M(n)) \rightarrow \mathbf{D}(G(p, n))$ is surjective. Thus, in particular, $\mathbf{D}(G(p, n))$ is commutative.

As an application, we examine how certain generalizations of the Radon transform and its dual, considered by the author [3] and Strichartz [14], intertwine the invariant differential operators on the manifolds $G(p, n)$. Specifically, fix $p$ and $q$ between 0 and $n-1$ and choose an integer $j$ with $\max (0, p+q-n) \leqq j \leqq \min (p, q)$. Define the transform $R(p, q, j)$ from functions on $G(p, n)$ to functions on $G(q, n)$ by

$$
R(p, q, j) f(\eta)=\int f(\xi) d \xi, \quad \eta \in G(q, n)
$$

when the integral is taken over all $p$-planes $\xi$ which intersect a given $q$-plane $\eta$ orthogonally in a $j$-dimensional plane. A result of Helgason on abstract Radon transforms [11] then enables us to show that for every $D \in \mathbb{Z}(M(n))$,

$$
R(p, q, j) \circ \mu_{p}(D)=\mu_{q}(D) \circ R(p, q, j)
$$

where $\mu_{p}$ and $\mu_{q}$ denote the projections of $\mathbf{Z}(M(n))$ onto $\mathbf{D}(G(p, n))$ and $\mathbf{D}(G(q, n))$, respectively. If $p+q=n-1, \mathbf{D}(G(p, n))$ and $\mathbf{D}(G(q, n))$ have the same number of algebraically independent generators [4] and in this special case one can find sets $\left\{E_{i}\right\}$ and $\left\{F_{i}\right\}$ of such generators of $\mathbf{D}(G(p, n))$ and $\mathbf{D}(G(q, n))$, respectively, such that

$$
R(p, q, 0) \circ E_{i}=F_{i} \circ R(p, q, 0) .
$$

This generalizes a well-known formula for the Radon transform and its dual (Lemma 2.1 of [9]).

The author is indebted to Professor S. Helgason for introducing him to the subject and for offering valuable insights. 


\section{The algebra $\mathrm{Z}(M(n))$}

The group $G=M(n)$ is isomorphic to the $(n+1) \times(n+1)$ matrix group

and it acts on $\mathbf{R}^{n}$ by

$$
\left\{\left(\begin{array}{ll}
k & V \\
0 & 1
\end{array}\right): k \in O(n), V \in \mathbf{R}^{n}\right\}
$$

$$
\left(\begin{array}{cc}
k & V \\
0 & 1
\end{array}\right) \cdot\left(\begin{array}{l}
Y \\
1
\end{array}\right)=k \cdot Y+V, \quad Y \in R^{n}
$$

Its Lie algebra $\mathfrak{g}$ is given by the set of matrices

$$
S=\left(\begin{array}{ll}
T & Z \\
0 & 0
\end{array}\right), \quad T \in \operatorname{so}(n), \quad Z \in \mathbf{R}^{n},
$$

so $(n)$ being the Lie algebra of $O(n)$. The adjoint representation $\mathrm{Ad}=\mathrm{Ad}_{G}$ of the group $G$ then satisfies

$$
A d\left(\begin{array}{ll}
k & V \\
0 & 1
\end{array}\right) \cdot\left(\begin{array}{ll}
T & Z \\
0 & 0
\end{array}\right)=\left(\begin{array}{cc}
k T k^{-1} & k \cdot Z-k T k^{-1} V \\
0 & 0
\end{array}\right) .
$$

As usual, let $E_{i j}$ denote the matrix $\left(\delta_{r i} \delta_{s j}\right)_{1 \geqq_{r}, \geqq_{n}+1}$ and put

$$
\begin{aligned}
X_{i j} & =E_{i j}-E_{j i} & & (1 \leqq i<j \leqq n) ; \\
U_{k} & =E_{k n+1} & & (1 \leqq k \leqq n) .
\end{aligned}
$$

These vectors form a basis of $\mathfrak{g}$.

Let $S(\mathrm{~g})$ be the symmetric algebra over $\mathrm{g}$ (consisting of polynomials in $\left\{X_{i j}, U_{k}\right\}$ with complex coefficients) and let $I(\mathrm{~g})$ be the algebra of $\operatorname{Ad}(G)$-invariant elements in $S(\mathrm{~g})$. As proved in [5], the symmetrization map

$$
\lambda: S(\mathfrak{g}) \rightarrow \mathbf{D}(G)
$$

is a linear bijection. We recall that for any basis $\left\{Z_{i}\right\}$ of $g$ and any $f \in C^{\infty}(G)$,

$$
\lambda(P) f(g)=\left\{P\left(\frac{\partial}{\partial t_{1}}, \ldots, \frac{\partial}{\partial t_{s}}\right) f\left(g \exp \left(\sum_{i} t_{i} Z_{i}\right)\right)\right\}_{\left(t_{i}\right)=0}, \quad P \in S(\mathrm{~g}),
$$

where $g \in G$. Since $\lambda$ commutes with the adjoint representation, its restriction to $I(\mathrm{~g})$ is a linear bijection onto $\mathbf{Z}(G)$. Although $\lambda$ is not multiplicative, we have by Lemma 4.2 of [4] that if $P_{1}, \ldots, P_{m}$ are algebraically independent generators of $I(\mathrm{~g})$, then $\lambda\left(P_{1}\right), \ldots, \lambda\left(P_{n}\right)$ are algebraically independent generators of $\mathbf{Z}(G)$. Thus to characterize $\mathbf{Z}(G)$ it suffices to produce a set of algebraically independent generators of $I(\mathrm{~g})$.

To describe these generators of $I(g)$ it is convenient to introduce some notation. Let $A=\left(a_{i j}\right)$ be any $N \times N$ matrix, and for each $1 \leqq k \leqq N$ let $1 \leqq i_{1}<$ 
$<i_{2}<\ldots<i_{k} \leqq N$ be a choice of $k$ indices in $\{1, \ldots, N\}$. For any such choice, let $D\left(i_{1}, i_{2}, \ldots, i_{k}\right)$ denote the $k \times k$ minor obtained from $A$ by choosing entries $a_{i j}$ when $i, j \in\left\{i_{1}, \ldots, i_{k}\right\}$. That is to say, $D\left(i_{1}, \ldots, i_{k}\right)=\operatorname{det}\left(a_{i j}\right)_{k \times k}\left(i, j \in\left\{i_{1}, \ldots, i_{k}\right\}\right)$. Also, let

$$
P_{k}(A)=\sum_{i_{1} \ldots, i_{k}} D\left(i_{1}, \ldots, i_{k}\right), \quad R_{k}(A)=\sum_{i_{1}, \ldots, i_{k-1}} D\left(i_{1}, \ldots, i_{k-1}, N\right)
$$

where the sums extend over all choices of the given indices.

Theorem 2.1. Consider the $(n+1) \times(n+1)$ skew-symmetric matrix with vector entries

$$
A=\left(\begin{array}{ccccc}
0 & X_{12} & \ldots & X_{1 n} & U_{1} \\
-X_{12} & 0 & \ldots & X_{2 n} & U_{2} \\
\cdot & \cdot & \ldots & \cdot & \cdot \\
\cdot & \cdot & \ldots & \cdot & \cdot \\
\cdot & \cdot & \ldots & \cdot & \cdot \\
-X_{1 n} & -X_{2 n} & \ldots & 0 & U_{n} \\
-U_{1} & -U_{2} & \ldots & -U_{n} & 0
\end{array}\right)
$$

For $1 \leqq j \leqq\left[\frac{1}{2}(n+1)\right]$ let $Q_{j} \in S(\mathrm{~g})$ be the sum $Q_{j}=R_{2 j}(A)$. (That is, $Q_{j}$ is the sum of the $2 j \times 2 j$ skew-symmetric minors of $A$ having vectors $U_{k}$ in the last row and column.) Then the polynomials $Q_{j}$ are algebraically independent generators of the algebra $I(\mathrm{~g})$.

For the proof we view $S(\mathrm{~g})$ as the algebra of complex-valued polynomial functions on the dual space $\mathfrak{g}^{*}$. Then $I(\mathfrak{g})$ is identified with the algebra $I_{0}\left(\mathfrak{g}^{*}\right)$ of polynomial functions on $\mathfrak{g}^{*}$ invariant under the coadjoint representation $\mathrm{Ad}^{*}$ of $G$ on $\mathfrak{g}^{*}$. Thus it suffices to obtain a set of generators for $I_{0}\left(\mathrm{~g}^{*}\right)$.

Consider now the linear bijection $\eta$ of $s o(n+1)$ onto $g^{*}$ given by

$$
\left(\begin{array}{cc}
X & U \\
-{ }^{t} U & 0
\end{array}\right) \rightarrow \eta_{X, U} \quad X \in s o(n), U \in R^{n}
$$

where, with $S$ as in (2)

$$
\eta_{X, U}(S)=\eta_{X, U}\left(\begin{array}{ll}
T & Z \\
0 & 0
\end{array}\right)=-\frac{1}{2} \operatorname{trace}\left(\begin{array}{cc}
X & U \\
-{ }^{t} U & 0
\end{array}\right)\left(\begin{array}{cc}
T & Z \\
-{ }^{t} Z & 0
\end{array}\right)=-\frac{1}{2} \operatorname{trace}(X T)+{ }^{t} U Z .
$$

Under this bijection, the coadjoint map $\operatorname{Ad}^{*}\left(\begin{array}{ll}k & V \\ 0 & 1\end{array}\right)$ on $\mathfrak{g}^{*}$ corresponds to the transformation of $s o(n+1)$ given by

$$
\begin{aligned}
& \left(\begin{array}{cc}
X & U \\
-{ }^{t} U & 0
\end{array}\right) \rightarrow\left(\begin{array}{ll}
k & V \\
0 & 1
\end{array}\right)\left(\begin{array}{cc}
X & U \\
-{ }^{t} U & 0
\end{array}\right)\left(\begin{array}{cc}
k^{-1} & 0 \\
{ }^{t} V & 1
\end{array}\right) . \\
= & \left(\begin{array}{cc}
k X k^{-1}-V^{t} U k^{-1}+k U^{t} V & k U \\
-{ }^{t} U k^{-1} & 0
\end{array}\right)=\left(\begin{array}{cc}
X^{\prime} & U^{\prime} \\
-{ }^{t} U^{\prime} & 0
\end{array}\right) .
\end{aligned}
$$


Indeed,

$$
\begin{gathered}
\left\{\operatorname{Ad}^{*}\left(\begin{array}{cc}
k & V \\
0 & 1
\end{array}\right) \cdot \eta_{X, v}\right\}\left(\begin{array}{cc}
T & Z \\
0 & 0
\end{array}\right)=\eta_{X, U}\left(\operatorname{Ad}\left(\begin{array}{cc}
k & V \\
0 & 1
\end{array}\right)^{-1} \cdot\left(\begin{array}{ll}
T & Z \\
0 & 0
\end{array}\right)\right) \\
=-\frac{1}{2} \operatorname{trace}\left(X k^{-1} T k\right)+{ }^{t} U k^{-1} T V+{ }^{t} U k^{-1} Z .
\end{gathered}
$$

On the other hand, by (8),

$$
\eta_{X^{\prime}, U^{\prime}}\left(\begin{array}{ll}
T & Z \\
0 & 0
\end{array}\right)=-\frac{1}{2} \operatorname{trace}\left(k X k^{-1} T-V^{t} U k^{-1} T+k U^{t} V T\right)+{ }^{t} U k^{-1} Z,
$$

which is easily seen to agree with (9). Thus, under the bijection $\eta$, the algebra $I_{0}\left(g^{*}\right)$ consists by (8) of the polynomial functions on $s o(n+1)$ invariant under the transformations

$$
\begin{gathered}
\left(\begin{array}{cc}
X & U \\
-{ }^{t} U & 0
\end{array}\right) \rightarrow\left(\begin{array}{ll}
k & 0 \\
0 & 1
\end{array}\right)\left(\begin{array}{cc}
X & U \\
-{ }^{t} U & 0
\end{array}\right)\left(\begin{array}{cc}
k^{-1} & 0 \\
0 & 1
\end{array}\right), \quad k \in O(n) \\
\left(\begin{array}{cc}
X & U \\
-{ }^{t} U & 0
\end{array}\right) \rightarrow\left(\begin{array}{cc}
I_{n} & V \\
0 & 1
\end{array}\right)\left(\begin{array}{cc}
X & U \\
-{ }^{t} U & 0
\end{array}\right)\left(\begin{array}{ll}
I_{n} & 0 \\
{ }^{t} V & 1
\end{array}\right),
\end{gathered}
$$

$I_{n}$ denoting the identity $n \times n$ matrix. Let $x_{i j}(1 \leqq i, j \leqq n)$ and $u_{k}(1 \leqq k \leqq n)$ denote the entry functions on the matrices $X \in s o(n)$ and $U \in \mathbf{R}^{n}$, respectively. Then the bijection $\eta$ identifies $g$ with the dual space $s o(n+1)^{*}$ via

$$
X_{i j} \rightarrow x_{i j}, \quad U_{k} \rightarrow u_{k}
$$

because $\eta_{X, U}\left(X_{i j}\right)=x_{i j}$ and $\eta_{X, U}\left(U_{k}\right)=u_{k}$. Since the transformations (ii) consist of simultaneous elementary row and column operations involving the last row and column of the skew-symmetric matrix $\left(\begin{array}{cc}X & U \\ -{ }^{t} U & 0\end{array}\right)$, it is clear from (10) and Lemma 2.2 at the end of this section that the polynomials $Q_{j}$ do indeed belong to $I(\mathrm{~g})$.

Next let $\left(\mathfrak{g}^{*}\right)^{\prime}$ be the subset of $\mathfrak{g}^{*}=s o(n+1)$ consisting of the matrices $\left(\begin{array}{cc}X & U \\ -{ }^{t} U & 0\end{array}\right)$ for which $|U|^{2}=u_{1}^{2}+\ldots+u_{n}^{2} \neq 0$. Then let $g_{0}^{*} \subset \mathrm{g}^{*}$ be the subspace of matrices

$$
\left(\begin{array}{rcc}
0 & 0 & u_{1} \\
0 & X^{\prime} & 0 \\
-u_{1} & 0 & 0
\end{array}\right) \quad u_{1} \in \mathbf{R}, \quad X^{\prime} \in \operatorname{so}(n-1) .
$$

Applying the transformations (i) and (ii) above, we see that the $\operatorname{Ad}^{*}(G)$-orbit of each point in $\left(\mathfrak{g}^{*}\right)^{\prime}$ intersects $\mathfrak{g}_{0}^{*}$. Consider the subgroup $G_{0} \subset G$ of elements $g \in G$ in (1) with $V=0$ and $k$ of the form

$$
\left(\begin{array}{rr} 
\pm 1 & 0 \\
0 & k_{1}
\end{array}\right) \quad k_{1} \in O(n-1)
$$


The action of $\operatorname{Ad}^{*}\left(G_{0}\right)$ on $\mathfrak{a}_{0}^{*}$ is given by

$$
\left(\begin{array}{ccc}
0 & 0 & u_{1} \\
0 & X^{\prime} & 0 \\
-u_{1} & 0 & 0
\end{array}\right) \rightarrow\left(\begin{array}{ccc}
0 & 0 & \pm u_{1} \\
0 & k_{1} X^{\prime} k_{1}^{-1} & 0 \\
\mp u_{1} & 0 & 0
\end{array}\right)
$$

Let $I_{G_{0}}\left(\mathfrak{g}_{0}^{*}\right)$ denote the corresponding algebra of $\mathrm{Ad}^{*}\left(G_{0}\right)$ - invariant polynomial functions on $\mathfrak{g}_{0}^{*}$. The restriction mapping $Q \rightarrow \bar{Q}=Q \mid \mathfrak{g}_{0}^{*}$ then maps $I_{0}\left(\mathfrak{g}^{*}\right)$ into $I_{G_{0}}\left(\mathfrak{g}_{0}^{*}\right)$. Since $\operatorname{Ad}^{*}(G) \cdot \mathfrak{g}_{0}^{*}$ contains $\left(\mathfrak{g}^{*}\right)^{\prime}$, which is dense in $\mathfrak{g}^{*}$, the restriction map is injective. Now because of Lemma 2.2 below, $I_{G_{0}}\left(g_{0}^{*}\right)$ is generated by $u_{I}^{2}$ and the algebraically independent polynomials $P_{2 k}\left(X^{\prime}\right)\left(1 \leqq k \leqq l=\left[\frac{n-1}{2}\right]\right)$, where as in (5) $P_{2 k}\left(X^{\prime}\right)$ is the sum of the $2 k \times 2 k$ skew-symmetric minors of $X^{\prime}$. It follows that $u_{1}^{2}, u_{1}^{2} P_{2}, \ldots, u_{1}^{2} P_{2 l}$ which coincide with $\bar{Q}_{1}, \bar{Q}_{2}, \ldots, \bar{Q}_{l+1}$ are algebraically independent so by the injectivity of the map $Q \rightarrow \bar{Q}$ the polynomials $Q_{1}, \ldots, Q_{t+1}$ are algebraically independent over $\mathbf{C}$.

It remains to prove that the algebra $I$ generated by $Q_{1}, \ldots, Q_{t+1}$ equals $I_{0}\left(\mathrm{~g}^{*}\right)$. Suppose there exists $Q \in I_{0}\left(g^{*}\right)$ not in $I$. Then $\bar{Q}$ is a polynomial

$$
\bar{Q}=S\left(u_{1}^{2}, P_{2}, \ldots, P_{2 l}\right)=S\left(\bar{Q}_{1}, \bar{Q}_{2} / \bar{Q}_{1}, \ldots, \bar{Q}_{l+1} / \bar{Q}_{1}\right)
$$

By the injectivity

$$
Q=S\left(Q_{1}, Q_{2} / Q_{1}, \ldots, Q_{l+1} / Q_{1}\right)=\frac{S_{1}\left(Q_{1}, \ldots, Q_{l+1}\right)}{Q_{1}^{k}},
$$

where $S_{1}$ is another polynomial. Since $Q \notin I$, we have $k \geqq 1$. By the algebraic independence of the $Q_{i}$, we may assume that the variable $t_{1}$ does not divide $S_{1}\left(t_{1}, \ldots, t_{l+1}\right)$. Write

$$
S_{1}\left(t_{1}, \ldots, t_{l+1}\right)=S^{\prime}\left(t_{2}, \ldots, t_{l+1}\right)+t_{1} S^{\prime \prime}\left(t_{1}, \ldots, t_{l+1}\right) \text {. }
$$

Then $S^{\prime}\left(t_{2}, \ldots, t_{l+1}\right) \neq 0$. We shall now show that there exists a complex matrix $\zeta_{0} \in \operatorname{so}(n+1, \mathrm{C})$ such that

$$
Q_{1}\left(\zeta_{0}\right)=0, \quad S^{\prime}\left(Q_{2}\left(\zeta_{0}\right), \ldots, Q_{l+1}\left(\zeta_{0}\right)\right) \neq 0 .
$$

For this consider the complex skew-symmetric matrices of the form

$$
\zeta=\left[\begin{array}{ccccccc}
0 & 0 & 0 & 0 & \ldots & 0 & 1 \\
0 & 0 & z_{23} & z_{24} & \ldots & z_{2 n} & i \\
0 & -z_{23} & 0 & z_{34} & \ldots & z_{3 n} & 0 \\
0 & -z_{24} & -z_{34} & 0 & \ldots & z_{4 n} & 0 \\
\vdots & \vdots & \vdots & \vdots & & & \\
0 & -z_{2 n} & -z_{3 n} & -z_{4 n} & \ldots & 0 & 0 \\
-1 & -i & 0 & 0 & \ldots & 0 & 0
\end{array}\right]
$$




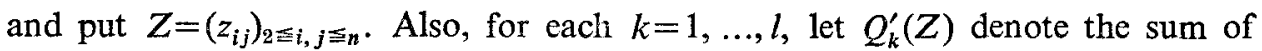
the $2 k \times 2 k$ skew-symmetric minors of $Z$ with entries from $z_{23}, z_{24}, \ldots, z_{2 n}$ in the first row and column. Then it is easy to see (because of a pairwise cancellation of minors) that

$$
Q_{1}(\zeta)=0, \quad Q_{k}(\zeta)=Q_{k-1}^{\prime}(Z) \quad(2 \leqq k \leqq l+1)
$$

Thus, $S^{\prime}\left(Q_{2}(\zeta), \ldots, Q_{l+1}(\zeta)\right)=S^{\prime}\left(Q_{1}^{\prime}(Z), \ldots, Q_{l}^{\prime}(Z)\right)$. However, the polynomial functions $Q_{1}^{\prime}, \ldots, Q_{l}^{\prime}$ were already seen to be algebraically independent over $\mathbf{C}$ so there exists $\zeta_{0}$ of the form (14) satisfying (13). This contradicts (12). Thus, $I=I_{0}\left(\mathrm{~g}^{*}\right)$.

To complete the proof of Theorem 2.1, we recall the following result $[12$, Ch. XII].

Lemma 2.2. Let $J$ be the algebra of polynomial functions on so(n) invariant under the adjoint action $X \rightarrow k X k^{-1}$ of $O(n)$. Then $J$ is generated by the polynomials $P_{2 k}\left(1 \leqq k \leqq\left[\frac{n}{2}\right]\right)$ where as in (5) $P_{2 k}(X)$ is the sum of the $2 k \times 2 k$ skew-symmetric minors of $X$. Moreover the $P_{2 k}$ are algebraically independent over $\mathbf{C}$.

Proof. Viewing each real $n \times n$ matrix $A$ as a linear transformation of $\mathbf{R}^{n}$, we have $P_{k}(A)=$ trace $\left(\Lambda^{k} A: A^{k} \mathbf{R}^{n} \rightarrow \Lambda^{k} \mathbf{R}^{n}\right)$. Thus $P_{k}(A)$ is certainly invariant under any change of basis transformation $A \rightarrow \tau A \tau^{-1}(\tau \in G L(n))$. (In fact, $\pm P_{k}(A)$ is the coefficient of $\lambda^{n-k}$ in the characteristic polynomial $\operatorname{det}\left(\lambda I_{n}-A\right)$.) Now each $X \in \operatorname{so}(n)$ is conjugate under $\operatorname{Ad}(O(n))$ to an element of the set $D$ of matrices

$$
\left(\begin{array}{rrrrrrr}
0 & s_{1} & & & & & \\
-s_{1} & 0 & & & & & \\
& & 0 & s_{2} & & & \\
& & -s_{2} & 0 & & & \\
& & & & & . & \\
& & & & & .
\end{array}\right) \text {. }
$$

Let $Q \in J$ and $\bar{Q}$ the restriction $Q \mid D$. Since $\operatorname{Ad}(O(n)) D=s o(n)$, the map $Q \rightarrow \bar{Q}$ is injective. Also, $\bar{Q}$ is invariant under the transformation $s_{i} \rightarrow \varepsilon_{i} s_{\sigma(i)}$ where $\varepsilon_{i}= \pm 1$ and $\sigma$ is any permutation (the Weyl group of so(n)). Thus, $\bar{Q}$ is a polynomial in the algebraically independent elementary symmetric polynomials of $s_{1}^{2}, \ldots, s_{t}^{2}$ $\left(t=\left[\frac{n}{2}\right]\right)$. However, these polynomials are just the restrictions to $D$ of the polynomials $P_{2 k}$. Thus, by the injectivity mentioned, the $P_{2 k}$ are algebraically independent and $Q$ is a polynomial in them.

The proof of Theorem 2.1 is now complete. 


\section{Central operators on other semidirect products}

Let $G$ be any real Lie group with Lie algebra $\mathfrak{g}$. If $\mathfrak{A}(\mathfrak{g})$ denotes the universal enveloping algebra of $g$ (with complex coefficients), then we have the identities [6]

$$
\mathfrak{U}(\mathfrak{g})=\mathfrak{U}\left(\mathfrak{g}^{\mathbf{C}}\right)=\mathbf{D}(G)=\mathbf{D}\left(G_{0}\right)
$$

where $G_{0}$ is the component of $G$ containing the identity and $\mathrm{g}^{\mathbf{C}}$ is the complexification of $\mathfrak{g}$. Letting $\mathfrak{z}(\mathrm{g})$ denote the center of $\mathfrak{Q}(\mathfrak{g})$, we also have

$$
\mathbb{Z}(G) \subseteq \mathbf{Z}\left(G_{0}\right)=\mathfrak{z}(\mathrm{g})=\mathfrak{z}\left(\mathrm{g}^{\mathrm{C}}\right)
$$

where $\mathbf{Z}\left(G_{0}\right)$ consists of the bi-invariant differential operators on $G_{0}$. Now extending each operator ad $X(X \in(\mathrm{g}))$ to a derivation of the symmetric algebra $S(\mathrm{~g})$, we define the polynomial algebra $I_{1}(\mathfrak{g})$ to be the set $\{P \in S(\mathfrak{g}) \mid \operatorname{ad}(X) P=0$ for all $X \in \mathfrak{g}\}$. Then $I_{1}(\mathrm{~g})$ coincides with the $\mathrm{Ad}\left(G_{0}\right)$-invariants in $S(\mathrm{~g}), I(\mathrm{~g}) \subseteq I_{1}(\mathrm{~g})$ and the symmetrization map $\lambda$ is a bijection of $I_{1}(\mathrm{~g})$ onto $z(\mathrm{~g})$.

Now take $G=M(n)$. Then $G_{0}$ is the semidirect product $S O(n) \times \mathbb{R}^{n}$. By the same proof as that of Theorem 2.1, with only the notation $k \in O(n)$ changed to $k \in S O(n)$, it can be shown that the algebra $I_{1}(\mathrm{~g})$ is also generated by the polynomials $Q_{1}, \ldots, Q_{t+1}$. Thus $I_{1}(\mathrm{~g})=I(\mathrm{~g})$ and so by $(16), z(\mathfrak{g})=\mathbb{Z}(G)$. By passing to the complexification, we obtain generators for $I_{1}\left(\mathrm{~g}^{\mathrm{C}}\right)$.

Theorem 3.1. For the Lie group $S O(n, \mathrm{C}) \times \mathbb{C}^{n}$ with Lie algebra $\mathfrak{g}^{\mathrm{C}}$ and basis vectors $X_{i j}, U_{k}$ as in (4), the algebra $I_{1}\left(\mathrm{~g}^{\mathrm{C}}\right)$ is generated by the algebraically independent polynomials $Q_{1}, \ldots, Q_{l+1}$ in Theorem 2.1 .

Next let $H$ be the connected general Poincaré group $S O_{0}(p, n-p) \times \mathbf{R}^{n}$, with Lie algebra $\mathfrak{H}=s o(p, n-p) \times \mathbb{R}^{n}$. $\mathfrak{H}$ has basis vectors $X_{r s}=E_{r s}-E_{s r}(1 \leqq r<s<p$, $p+1 \leqq r<s \leqq n), Y_{r s}=E_{r s}+E_{s r}(1 \leqq r \leqq p, p+1 \leqq s \leqq n)$, and $U_{k}=E_{k, n+1}(1 \leqq k \leqq n)$. The complex Lie algebra $\mathfrak{g}^{\mathrm{C}}$ is canonically isomorphic to the complexification $\mathfrak{S}^{\mathrm{C}}$ via the map

$$
\varphi:\left(\begin{array}{ccc}
X_{1} & X_{2} & U_{1} \\
-{ }^{t} X_{2} & X_{3} & U_{2} \\
0 & 0 & 0
\end{array}\right) \rightarrow\left(\begin{array}{ccc}
X_{1} & i X_{2} & U_{1} \\
i^{t} X_{2} & X_{3} & -i U_{2} \\
0 & 0 & 0
\end{array}\right)
$$

Here $X_{1} \in \operatorname{so}(p, \mathbf{C}), X_{3} \in s o(n-p, \mathbf{C}), X_{2}$ is any complex $p \times(n-p)$ matrix, $U_{1} \in \mathbf{C}^{p}$, and $U_{2} \in C^{n-p}$. From Theorem 3.1 and (17) it follows that the polynomials $\left.Q_{j}{ }^{\prime \prime}\left\{\varphi\left(X_{r s}\right)\right\},\left\{\varphi\left(U_{k}\right)\right\}\right)\left(1 \leqq j \leqq\left[\frac{1}{2}(n+1)\right]\right)$ constitute a set of algebraically independent 
generators of $I\left(\mathfrak{H}^{\mathrm{C}}\right)=I(\mathfrak{H})$. Now

$$
\begin{aligned}
& \varphi\left(X_{r s}\right)= \begin{cases}X_{r s} & 1 \leqq r<s \leqq p \text { or } p+1 \leqq r<s \leqq n \\
i Y_{r s} & 1 \leqq r \leqq p, p+1 \leqq s \leqq n ;\end{cases} \\
& \varphi\left(U_{k}\right)=\left\{\begin{array}{rl}
U_{k} & 1 \leqq k \leqq p \\
-i U_{k} & p+1 \leqq k \leqq n .
\end{array}\right.
\end{aligned}
$$

From Theorem 2.1 we obtain the generators of the invariant algebra $I(\mathfrak{S})$

Theorem 3.2. Consider the $(n+1) \times(n+1)$ matrix with vector entries

$$
B=\left[\begin{array}{ccccccccc}
0 & X_{12} & \ldots & X_{1 p} & Y_{1, p+1} & Y_{1, p+2} & \ldots Y_{1 n} & U_{1} \\
-X_{12} & 0 & \ldots & X_{2 p} & Y_{2, p+1} & Y_{2, p+2} & \ldots Y_{2 n} & U_{2} \\
\vdots & \vdots & & \vdots & \vdots & \vdots & \vdots & \vdots \\
-X_{1 p} & -X_{2 p} & \ldots & 0 & Y_{p, p+1} & Y_{p, p+2} & \ldots Y_{p n} & U_{p} \\
Y_{1, p+1} & Y_{2, p+1} & \ldots & Y_{p, p+1} & 0 & X_{p+1, p+2} & \ldots X_{p+1, n} & U_{p+1} \\
Y_{1, p+2} & Y_{2, p+2} & \ldots & Y_{p, p+2} & -X_{p+1, p+2} & 0 & \ldots X_{p+2, n} & U_{p+2} \\
\vdots & \vdots & & \vdots & \vdots & \vdots & & \vdots & \vdots \\
Y_{1 n} & Y_{2 n} & \ldots & Y_{p n} & -X_{p+1, n} & -X_{p+2, n} & \ldots & 0 & U_{n} \\
-U_{1} & -U_{2} & \ldots & -U_{p} & U_{p+1} & U_{p+2} & \ldots U_{n} & 0
\end{array}\right] .
$$

Then the polynomials $R_{2 j}(B)\left(1 \leqq j \leqq\left[\frac{n+1}{2}\right]\right)$ are algebraically independent generators of $I(\mathfrak{S})$.

In fact, $Q_{j}\left(\left\{\varphi\left(X_{k l}\right)\right\},\left\{\varphi\left(U_{k}\right)\right\}\right)=R_{2 j}(B)$.

As an example, let $n=4$ and $p=3$. Then computing by means of Theorem 3.2, the algebra of bi-invariant differential operators on the connected Poincare group $S O_{0}(3,1) \times R^{4}$ can be shown to have two algebraically independent generators, these being the images under the symmetrization $\lambda$ of the second order polynomial $U_{1}^{2}+U_{2}^{2}+U_{3}^{2}-U_{4}^{2}$ and the fourth order polynomial $\left(X_{12} U_{3}-X_{13} U_{2}+X_{23} U_{1}\right)^{2}-$ $\left(X_{12} U_{4}+X_{14} U_{2}-Y_{24} U_{1}\right)^{2}-\left(X_{13} U_{4}+Y_{14} U_{3}-Y_{34} U_{1}\right)^{2}-\left(X_{23} U_{4}+Y_{24} U_{3}-Y_{34} U_{2}\right)^{2}$. This result has been obtained previously by Varadarajan (see [15]).

\section{Projections on Grassmamians and applications to Radon transforms}

In this section $G$ will denote the Euclidean motion group $M(n)$. For $0 \leqq p \leqq n-1$, let $E_{p}$ denote the subspace spanned by the first $p$ basis elements of $\mathbf{R}^{n}\left(E_{p}=0\right.$ if $p=0)$, and let $H$ be the subgroup of $G$ leaving $E_{p}$ fixed. Then $H=M(p) \times O(n-p)$ and $G / H$ is the affine Grassmann manifold $G(p, n)$ of $p$-planes in $\mathbf{R}^{n}$. Denote by 
$\mathbf{D}(G / H)$ the algebra of differential operators on $G / H$ which are invariant under the $G$-action. If $\pi: G \rightarrow G / H$ is the natural projection, we have a homomorphism $\mu$ of $\mathbf{Z}(G)$ into $\mathbf{D}(G / H)$ given by

$$
(\mu(D) f) \circ \pi=D(f \circ \pi)
$$

for $D \in \mathbf{Z}(G)$ and $f \in C^{\infty}(G / H)$ ([7]). We note that (19) also defines $\mu(D) \in \mathbf{D}(G / H)$ for any $\mathbf{D} \in \mathbf{D}(G)$ which is invariant under right translations by all $h \in H$.

Theorem 4.1. $\mu$ maps $\mathbf{Z}(G)$ onto $\mathbf{D}(G / H)$.

Remark. Since $\mathbf{Z}(G)$ is commutative, so is $\mathbf{D}(G / H)$ by Theorem 4.1. The commutativity of $\mathbf{D}(G / H)$ is also a consequence of the fact that the pair $(G, H)$ is a symmetric pair ([1], [13]).

For the proof of Theorem 4.1, we decompose the Lie algebra $\mathfrak{g}$ of $G$ into a direct sum of $\mathfrak{H}$, the Lie algebra of $H$, and an $\operatorname{Ad}(H)$-invariant subspace $\mathfrak{M}$. Since $H$ consists of the matrices

$$
h=\left(\begin{array}{lll}
a & 0 & V \\
0 & b & 0 \\
0 & 0 & 1
\end{array}\right) \quad \underset{V \in \mathbb{R}^{p}}{a \in O(p), b \in O(n-p),}
$$

we define $\mathfrak{M} \subseteq \mathfrak{g}$ as the subspace of matrices

$$
T=\left(\begin{array}{rrr}
0 & Y & 0 \\
{ }^{t} Y & 0 & W \\
0 & 0 & 0
\end{array}\right) \begin{aligned}
& Y \text { any real } p \times(n-p) \text { matrix } \\
& W \in \mathbf{R}^{n-p}
\end{aligned}
$$

Then $\mathfrak{g}=\mathfrak{S} \oplus \mathfrak{M}$ and since

$$
\operatorname{Ad}(h) T=\left(\begin{array}{ccc}
0 & a Y b^{-1} & 0 \\
-b^{t} Y a^{-1} & 0 & b \cdot W+b^{t} Y a^{-1} V \\
0 & 0 & 0
\end{array}\right)
$$

$\mathfrak{M}$ is $\operatorname{Ad}(H)$-invariant. Now for every $P \in S(\mathfrak{g})$, there exists a unique polynomial $\bar{P} \in S(\mathfrak{M})$ such that $P-\bar{P} \in S(\mathfrak{g}) \mathfrak{H}$. Let $I(\mathfrak{M})$ be the algebra of $\mathrm{Ad}(H)$-invariants in $S(\mathfrak{M})$. Then the map $P \rightarrow \bar{P}$ takes $I(\mathfrak{g})$ into $I(\mathfrak{M})$. Since the pair $(G, H)$ is reductive [4], it suffices by [10, Chapter II, Proposition 5.32] to prove that the map $P \rightarrow \bar{P}$ takes $I(\mathfrak{g})$ onto $I(\mathfrak{M})$. Now $\mathfrak{M}$ has basis vectors $X_{i j}(1 \leqq i \leqq p, p+1 \leqq j \leqq n)$ and $U_{k}(p+1 \leqq k \leqq n)$. We recall the characterization of $I(\mathfrak{M})$ in terms of these basis vectors [4].

Lemma 4.2. Consider the $(p+1) \times(n-p)$ matrix with vector entries

$$
C=\left(\begin{array}{lll}
U_{p+1} & \ldots & U_{n} \\
X_{1, p+1} & \ldots & X_{1, n} \\
X_{p, p+1} & \ldots & X_{p, n}
\end{array}\right)
$$


For $1 \leqq k \leqq \min (p+1, n-p)$ let $T_{k} \in S(\mathfrak{M})$ be the sum of the squares of the $k \times k$ minors of $C$ having vectors $U_{j}$ in the first row:

$$
T_{k}=\sum_{\substack{p+1 \leqq i_{1}<\ldots<i_{k} \leqq n \\
1 \leqq j_{1}<\ldots<j_{k-1} \leqq p}} \operatorname{det}^{2}\left(\begin{array}{ll}
U_{i_{1}} & \ldots U_{i_{k}} \\
X_{j_{1}, i_{1}} & \ldots X_{j_{1}, i_{k}} \\
& \ldots \\
X_{j_{k-1}, i_{1}} \ldots X_{j_{k-1}, i_{k}}
\end{array}\right) .
$$

Then the polynomials $T_{k}$ are algebraically independent generators of $I(M)$.

We will show that for the generators $Q_{k}$ of $I(\mathfrak{g})$ in Theorem 2.1, $\bar{Q}_{k}=T_{k}$ when $1 \leqq k \leqq \min (p+1, n-p)$. Since the map $P \rightarrow \bar{P}$ is a homomorphism, this will show that it is surjective from $I(\mathfrak{g})$ to $I(\mathfrak{M})$.

For this purpose it is convenient to identify $S(\mathfrak{g})$ and $S(\mathfrak{M})$ with the algebras of polynomial functions on the dual spaces $\mathfrak{g}^{*}$ and $\mathfrak{M}^{*}$, respectively. Thus, as in the proof of Theorem 2.1, $I(\mathfrak{g})=I_{0}\left(\mathfrak{g}^{*}\right)$. By the same token, $I(\mathfrak{M})$ is identified with the algebra $I_{H}\left(\mathfrak{M}^{*}\right)$ of polynomial functions $Q$ on $\mathfrak{M}^{*}$ invariant under the co-isotropy representation $\operatorname{Ad}_{G}^{*}(H)$ on $\mathfrak{M}^{*}$

$$
Q\left(\operatorname{Ad}^{*}(h) f\right)=Q(f) \quad h \in H, f \in \mathfrak{m}^{*} .
$$

By letting each $f \in \mathfrak{M}^{*}$ be identically zero on $\mathfrak{H}$ we may assume $\mathfrak{M}^{*} \subset \mathfrak{g}^{*}$. If $P \in S(\mathfrak{g})$, $\bar{P}$ then coincides with the restriction $P \mid \mathfrak{M}^{*}$. Obviously if $P \in I\left(\mathfrak{g}^{*}\right)$ then $\bar{P} \in I_{H}\left(\mathfrak{M}^{*}\right)$. Under the bijection $(7)$ of $s o(n+1)$ onto $\mathfrak{g}^{*}$, the subspace $\mathfrak{M}^{*} \subset \mathfrak{g}^{*}$ corresponds to the subspace of skew-symmetric matrices of the form

$$
A=\left(\begin{array}{rrr}
0 & X & 0 \\
-t & 0 & U \\
0 & { }^{-t} U & 0
\end{array}\right) \quad \begin{aligned}
& X \text { any real } p \times(n-p) \text { matrix, } \\
& U \in \mathbf{R}^{n-p}
\end{aligned}
$$

From this we also obtain a linear bijection of $\mathfrak{M}^{*}$ onto the space $M_{p+1, n-p}$ of real $(p+1) \times(n-p)$ matrices as follows:

$$
A \rightarrow\left(\begin{array}{c}
{ }^{t} U \\
X
\end{array}\right)=\left(\begin{array}{ccc}
u_{p+1} & \ldots & u_{n} \\
x_{1, p+1} & \ldots & x_{1 n} \\
x_{p, p+1} & \ldots & x_{p n}
\end{array}\right)
$$

By means of the transpose map, $\mathfrak{M}$ corresponds to the dual space $M_{p+1, n-p}^{*}$, and by (10) the basis vectors of $\mathfrak{M}$ correspond to the entry functions of $M_{p+1, n-p}$ via

$$
\begin{array}{rl}
X_{i j} \rightarrow x_{i j} & 1 \leqq i \leqq p, p+1 \leqq j \leqq n \\
U_{k} \rightarrow u_{k} & p+1 \leqq k \leqq n .
\end{array}
$$

Thus the polynomials $T_{k}$ are polynomial functions on the space $M_{p+1, n-p}$, just as the $Q_{k}$ are polynomial functions on $s o(n+1)$. Moreover, it is easy to see that for 
any matrix $A$ of the form (21),

$$
Q_{k}(A)=T_{k}\left(\begin{array}{c}
t \\
X
\end{array}\right) \quad k=1, \ldots, \min (p+1, n-p)
$$

Thus it follows that $\bar{Q}_{k}=T_{k}$, and this proves Theorem 4.1.

Remark. If $k>\min (p+1, n-p)$, then $Q_{k} \mid \mathfrak{M}^{*}=0$.

Corollary 4.3. The operators $\mu\left(\lambda\left(Q_{1}\right)\right), \ldots, \mu\left(\lambda\left(Q_{m}\right)\right)(m=\min (p+1, n-p))$ are algebraically independent generators of $\mathbf{D}(G(p, n))$.

Proof. By $\left[4\right.$, Lemma 4.2] the operators $\mu\left(\lambda\left(T_{1}\right)\right), \ldots, \mu\left(\lambda\left(T_{m}\right)\right)$ are algebraically independent generators of $\mathbf{D}(G(p, n))$. Since $\bar{Q}_{k}=T_{k}(1 \leqq k \leqq m)$, we have

$$
\mu\left(\lambda\left(Q_{k}\right)\right)=\mu\left(\lambda\left(T_{k}\right)\right)+\text { lower order terms }
$$

([7]). Now suppose $P=\sum a_{n_{1}, \ldots, n_{m}} x_{1}^{n_{1}} \ldots x_{m}^{n_{m}}$ is a nonzero polynomial such that the differential operator $D=P\left(\mu\left(\lambda\left(Q_{1}\right)\right), \ldots, \mu\left(\lambda\left(Q_{m}\right)\right)\right)=0$. Let $D^{\prime}=$ $P\left(\mu\left(\lambda\left(T_{1}\right)\right), \ldots, \mu\left(\lambda\left(T_{m}\right)\right)\right)$. Then $D^{\prime} \neq 0$ and by $(24)$, order $\left(D^{\prime}-D\right)<\operatorname{order}\left(D^{\prime}\right)$. This yields order $\left(D^{\prime}\right)<\operatorname{order}\left(D^{\prime}\right)$, a contradiction. Thus $\mu\left(\lambda\left(Q_{1}\right)\right), \ldots, \mu\left(\lambda\left(Q_{m}\right)\right)$ are algebraically independent. Next let $D \in \mathbf{D}(G(p, n))$. Then we may write $D=P\left(\mu\left(\lambda\left(T_{1}\right)\right), \ldots, \mu\left(\lambda\left(T_{m}\right)\right)\right)$ for some polynomial $P$. Setting

$$
D_{1}=P\left(\mu\left(Q_{1}\right), \ldots, \mu\left(\lambda\left(Q_{m}\right)\right)\right)
$$

we have $D=D_{1}+D_{2}$, where order $\left(D_{2}\right)<\operatorname{order}(D)$. The corollary follows by induction on the order of $D_{2}$.

Now fix a value of $q$ between 0 and $n-1$, and fix $j$ between $\max (0, p+q-n)$ and $\min (p, q)$. We consider a generalization of the Radon transform and its dual, due to Strichartz [14], from functions on $G(p, n)$ to functions on $G(q, n)$. For a fixed $q$-plane $\eta$, let $\hat{\eta}$ be the set of all $p$-planes $\xi$ intersecting $\eta$ orthogonally in a $j$-dimensional plane. Then $\hat{\eta}$ is a closed submanifold of $G(p, n)$ and there exists a canonical measure $\mathrm{d} \mu(\xi)$ on $\hat{\eta}$ invariant under all Euclidean motions preserving $\eta$ (cf. below). For any suitable function $f$ on $G(p, n)$, the transform $R(p, q, j) f$ is a function on $G(q, n)$ defined by

$$
R(p, q, j) f(\eta)=\int_{\eta} f(\xi) d \mu(\xi), \quad \eta \in G(q, n) .
$$

For our purposes it is necessary to formulate this integral transform in terms of homogeneous spaces in duality. Let $e_{1}, \ldots, e_{n}$ be the usual basis of $R^{n}$, let $E_{p}$ be as before the span of $e_{1}, \ldots, e_{p}$ and let $E_{q}$ be the span of $e_{p-j+1}, \ldots, e_{p-j+q}$. Then $E_{p}$ and $E_{q}$ meet orthogonally in a $j$-dimensional plane. If $H_{p}$ and $H_{q}$ are the respective subgroups of $G$ leaving $E_{p}$ and $E_{q}$ invariant, then $H_{p}$ consists of the $(n+1) \times(n+1)$ 
matrices $h$ in (20) while $H_{q}$ consists of the $(n+1) \times(n+1)$ matrices

$$
\left(\begin{array}{llll}
a^{\prime} & 0 & 0 & 0 \\
0 & b^{\prime} & 0 & V^{\prime} \\
0 & 0 & c^{\prime} & 0 \\
0 & 0 & 0 & 1
\end{array}\right), \quad \begin{aligned}
& a^{\prime} \in O(p-j), b^{\prime} \in O(q), \\
& c^{\prime} \in O(n+j-p-q), V^{\prime} \in \mathbf{R}^{q} .
\end{aligned}
$$

Also, $G(p, n)=G / H_{p}$ and $G(q, n)=G / H_{q}$.

Proposition 4.4. The manifolds $G / H_{p}$ and $G / H_{q}$ are homogeneous spaces in duality. That is to say, the groups $G, H_{p}, H_{q}$, and $H=H_{p} \cap H_{q}$ satisfy the following properties:

(i) They are all unimodular.

(ii) If $h_{p} \in H_{p}$ satisfies $h_{p} H_{q} \subset H_{q} H_{p}$, then $h_{p} \in H_{q}$.

(iii) $H_{p} H_{q}$ is a closed subset of $G$.

The proof is straightforward and shall be omitted. (See [9] for the assumptions underlying homogeneous spaces in duality.)

The transform $R(p, q, j)$ is then the integral transform associated with the double fibration

$$
G / \dot{H}_{p}^{G / H} \bar{G} / H_{a}
$$

That is to say, if $\eta=g \cdot E_{q}(g \in G)$, then $\hat{\eta}=\left\{g h_{q} \cdot E_{p} \mid h_{q} \in H_{q}\right\}$ and

$$
R(p, q, j) f(\eta)=\int_{H_{q} / \boldsymbol{H}} f\left(g h_{q} \cdot E_{p}\right) d\left(h_{q}\right)_{H}
$$

where $d\left(h_{q}\right)_{H}$ is the $H_{q}$-invariant measure on $H_{q} / H$. (See [9].) A result of Helgason [11, Proposition 4.1] states that an integral transform associated with a double fibration such as (26) intertwines the $G$-invariant differential operators in $G / H_{p}$ and $G / H_{q}$ arising from operators in $\mathbf{Z}(G)$.

Proposition 4.5. For any $D \in \mathbb{Z}(G)$, let $\mu_{p}(D)$ and $\mu_{q}(D)$ be the projections of $D$ on $G / H_{p}$ and $G / H_{q}$, respectively, as in (19). Then for any $f \in C_{c}^{\infty}(G(p, n))$,

$$
R(p, q, j)\left(\mu_{p}(D) f\right)=\mu_{q}(D)(R(p, q, j) f) .
$$

Note that by Theorem 4.1, (27) is a statement about how $R(p, q, j)$ intertwines all $G$-invariant differential operators on $G / H_{p}$ and $G / H_{q}$.

Finally we consider the case $q=n-p-1$. By Corollary 4.3 , the algebras $\mathbf{D}(G(p, n))$ and $\mathbf{D}(G(q, n))$ have the same number of algebraically independent generators, these being $E_{i}=\mu_{p}\left(\lambda\left(Q_{i}\right)\right)$ and $F_{i}=\mu_{q}\left(\lambda\left(Q_{i}\right)\right)(1 \leqq i \leqq \min (p+1, q+1))$ respectively. (27) shows how $R(p, q, j)$ intertwines these generators:

$$
R(p, q, j) \circ E_{i}=F_{i} \circ R(p, q, j) .
$$


When $j=0$, the transform $R(p, q, 0)$ is injective (and in fact was inverted explicitly in [3]), and (28) generalizes the well known relations for the Radon transform $R(=R(0, n-1,0))$ and its dual $R^{t}(=R(n-1,0,0))$ on $\mathbf{R}^{n}$ :

$$
R(L f)=\square(R f), \quad R^{t}(\square \varphi)=L R^{t} \varphi,
$$

for all $f \in C_{c}^{\infty}\left(R^{n}\right), \varphi \in C_{c}^{\infty}(G(n-1, n))$, where $L$ is the Laplacian on $\mathbf{R}^{n}$ and $\square$ is the Laplacian on the fibers of the vector bundle $G(n-1, n)([8],[9])$.

\section{References}

1. Duflo, M., Opérateurs invariants sur un espace symétrique, C.R. Acad. Sci. Paris Sér, A 289 (1979), 135-137.

2. Gonzalez, F., Ph. D. Thesis, M.I.T. (1984).

3. Gonzalez, F., Radon transforms on Grassmann manifolds, J. Funct. Anal. (to appear).

4. Gonzalez, F. and Helgason, S., Invariant differential operators on Grassmann manifolds, Adv. in Math. 60 (1986), 81-91.

5. Harish-Chandra, On representations of Lie algebras, Ann. Math. 50 (1949), 900-915.

6. Harish-Chandra, The characters of semi-simple Lie groups, Trans. Amer. Math. Soc. 83 (1956), 98-163.

7. Helgason, S., Differential operators on homogeneous spaces, Acta Math. 102 (1959), 239299.

8. Helgason, S., The Radon transform on Euclidean spaces, compact two-point homogeneous spaces, and Grassmann manifolds, Acta Math. 113 (1965), 153-180.

9. Helgason, S., The Radon Transform, Birkhäuser, Boston, 1980.

10. Helgason, S., Groups and Geometric Analysis, Academic Press, 1984.

11. Helgason, S., Some results on Radon transforms, Huygens' principle, and X-ray transforms, Contemporary Mathematics, Vol. 63 (Integral Geometry), Amer. Math. Soc. 1986.

12. КоваYAshi, S. and Nomizu, K., Foundations of Differential Geometry, II, Wiley (Interscience), New York, 1969.

13. Lichnerowicz, A., Opérateurs đifférentiels sur un espace symétrique, C.R. Acad. Sci. Paris 257 (1963), 3548-3550.

14. Strichartz, R., Harmonic analysis on Grassmannian bundles, Trans. Amer. Math. Soc., 296 (1986), 387-409.

15. TAKIFF, S., Invariant polynomials on Lie algebras of inhomogeneous unitary and special orthogonal groups, Trans. Amer. Math. Soc. 170 (1972), 221-230.

Received March 3, 1987

Department of Mathematics and Statistics

Case Western Reserve University

Cleveland, Ohio 44106

U.S.A.

Present Address: Department of Mathematics

Tufts University

Medford, Massachusetts 02155

U.S.A. 